\title{
Mess-, Automatisierungs- und Robotertechnik
}

\section{G. Schitter OVE}

Online publiziert am 10. Juli 2015

(C) Springer Verlag Wien 2015

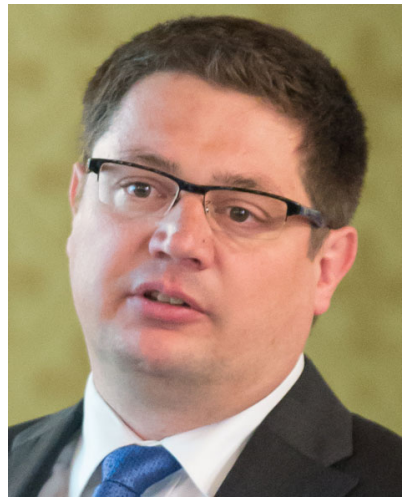

Univ.-Prof. Dipl.-Ing. Dr.sc.techn. Georg Schitter Dipl. NDS ETHZ
Anlässlich der Gründung der Gesellschaft für Mess-, Automatisierungs- und Robotertechnik (GMAR) im Jänner 2015 stellen die Beiträge in diesem Sonderheft der e\&i inhaltlich die drei Fachbereiche der GMAR vor:

- Messtechnik und Senso rik,

- Automatisierungs-, Regelungstechnik und Mechatronik sowie

- Robotik.

Als österreichische Plattform ist die GMAR der Ansprechpartner für sämtliche Belange der Messtechnik, Automatisierungs- und Regelungstechnik sowie Robotik als wesentliche Zukunftstechnologien und Treiber für nachhaltiges wirtschaftliches Wachstum in Österreich. Die GMAR ist eine Fachgesellschaft unter der Schirmherrschaft der drei Trägervereine Österreichischer Verband für Elektrotechnik (OVE), Österreichische Computer Gesellschaft (OCG) und Österreichischer Ingenieur- und ArchitektenVerein (ÖIAV) und versteht sich als gesamthafte Vertretung der in diesen Bereichen tätigen oder daran interessierten Unternehmen, Forschungseinrichtungen, wissenschaftlichen Institutionen, Bildungseinrichtungen und Personen in Österreich.

Insgesamt umfasst dieses e\&i-Schwerpunktheft sieben Beiträge, bestehend aus zwei wissenschaftlichen Beiträgen zum Fachbereich Messtechnik und Sensorik, drei wissenschaftlichen Fachartikeln aus dem Fachbereich Automatisierungs-, Regelungstechnik und Mechatronik sowie zwei Übersichtsartikeln jeweils zu den industriellen und akademischen Forschungsaktivitäten in Österreich im Fachbereich Robotik.

Der erste Beitrag aus dem Fachbereich Messtechnik und Sensorik von Almir Talic, Samir Cerimovic, Franz Kohl, Artur Jachimowicz, Thilo Sauter, Thomas Voglhuber-Brunnmaier und Bernhard Jakoby mit dem Titel "Miniaturisierte thermische Fluidsensoren - Strömungsund thermische Leitfähigkeitsensoren" gibt einen sehr guten Überblick zum aktuellen Stand von mikrotechnisch hergestellten thermischen Fluidsensoren, vor allem zu kalorimetrischen Strömungssensoren (KSS) und thermischen Leitfähigkeitssensoren (TLS), mit einigen Fallbeispielen aus den Forschungsarbeiten der Autoren.

Im zweiten Beitrag mit dem Titel „Einsatz von Magnetfeldsensoren in der zerstörungsfreien Materialprüfung und Qualitätssicherung" widmen sich Patrick Hölzl, Florian Dietachmayr, Johannes Atzlesberger und Bernhard Zagar dem Einsatz von drei unterschiedlichen magnetischen Sensoren in der Materialprüfung. Diese Sensoren unterscheiden sich hauptsächlich im geometrischen Aufbau
(Punkt-, Linien- und Flächensensor) bzw. im Messprinzip (GMR und Faraday-Effekt). Die Aufnahme eines Magnetfeldprofils mit Einzelsensoren resultiert in einer vergleichsweise langen Messdauer, wohingegen der beschriebene Zeilensensor eine wesentlich schnellere Messung ermöglicht. Mithilfe eines Flächensensors, basierend auf dem Faraday-Effekt, der sowohl für stationäre Probleme als auch für transiente Vorgänge verwendet werden kann, wird ein instantaner Scan des Magnetfeldprofils durchgeführt.

Georg Stettinger, Martin Benedikt, Martin Horn und Josef Zehetner diskutieren in ihrem Beitrag mit dem Titel „Modellbasierte Echtzeit-Co-Simulation: Überblick und praktische Anwendungsbeispiele" aus dem Fachbereich Automatisierungs-, Regelungstechnik und Mechatronik Ergebnisse einer Kooperation zwischen Industrie und Akademia zu Herausforderungen der Co-Simulation in der Praxis. Kommunikationsbedingte Latenzzeiten und verrauschte Koppelgrößen stellen beim Einbinden von realen Hardwarekomponenten die größten Herausforderungen bei der Co-Simulation dar. Zur Behandlung dieser Störungen wird ein modellbasierter Kopplungsansatz vorgestellt, wobei mittels Prädiktion von Koppelgrößen Störeinflüsse deutlich reduziert werden können. Die Effektivität dieses Kopplungsansatzes wird an einem Labormodell demonstriert.

Der zweite Beitrag aus dem Fachbereich Automatisierungs-, Regelungstechnik und Mechatronik mit dem Titel „Flache konzentriertparametrische Systeme, Theorie und Praxis" von Bernd Kolar und Kurt Schlacher gibt einen sehr guten Überblick über die Anwendung der Flachheit für Trajektorienplanung und Regelung für verschiedene Systemklassen. Die Konzepte werden an zwei theoretischen Beispielen und einem praktischen Versuchsaufbau demonstriert. Nach kurzer Einführung werden in diesem Beitrag die Grundlagen des Reglerentwurfs, der Trajektorienplanung und deren Stabilisierung gezeigt. Diese werden dann auf zwei einfache Beispiele, ein Pendel mit Kreisel und ein Einachsenmodell eines Fahrzeugs, angewandt. Als weitere illustrative Beispiele folgen die Trajektorienplanung und nichtlineare Regelung für einen Brückenkran, für die theoretische Ergebnisse, Simulationen und Laborversuche präsentiert werden.

Florian Schausberger, Andreas Steinboeck und Andreas Kugi präsentieren im dritten Beitrag aus dem Fachbereich Automatisierungs-, Regelungstechnik und Mechatronik mit dem Titel „Modellbasierte Optimierung und Regelung der Produktkontur beim Warmwalzen" ein optimierungsbasiertes Verfahren zur Beseitigung der beim Warmwalzen auftretenden Abweichungen der Produktkontur. Die vorgestellte Methode zur Schätzung der Kontur einer Walztafel ermöglicht eine präzise Bestimmung der Walztafelkontur. Durch das mathematische Modell der Konturentwicklung kann eine genaue Abbildung der Verhältnisse im Walzspalt im Hinblick auf die resultierende Kontur erreicht werden. Die Kombination der Konturschätzung und des Modells zur Säbelbildung stellt deshalb einen vielver-

Schitter, G., Institut für Automatisierungs- und Regelungstechnik, Technische Universität Wien, Gußhausstraße 25-29/E376, 1040 Wien, Österreich

(E-Mail: georg.schitter@tuwien.ac.at) 
sprechenden Ansatz zur Beseitigung vorhandener Säbel und somit zur Steigerung der Produktqualität dar.

Der erste Beitrag aus dem Fachbereich Robotik mit dem Titel „Robotik in Industrie und Forschung - ein Überblick über die Robotik landschaft in Österreich" mit Fallbeispielen von Verena Kriegl und Paolo Ferrara zeigt, dass Robotik längst Einzug in die österreichische Industrie gefunden hat und mittlerweile fester Bestandteil dieser geworden ist. In Österreich gibt es rund 70 Unternehmen, die sich direkt oder indirekt mit dem Thema Robotik beschäftigen, wobei die Themen breit gefächert sind: Die Bandbreite reicht von Steuerungslösungen für Industrieroboter, über frei navigierende Transportroboter, bis hin zu massentauglichen Haushaltsrobotern. Damit sich die Robotik-Industrie in Zukunft weiterentwickeln kann, ist eine enge Verzahnung von Forschung und Industrie notwendig, und es bedarf mutiger Pilotkunden, die dafür offen sind, als Vorreiter neue Entwicklungen einzusetzen.

Der zweite Beitrag aus dem Fachbereich Robotik von Michael Hofbaur, Andreas Müller, Justus Piater, Bernhard Rinner, Gerald Steinbauer, Markus Vincze und Christian Wögerer mit dem Titel "Making Better Robots - Beiträge Österreichs zur europäischen Robotics Research Roadmap" stellt Forschungsinstitutionen und Projekte in Österreich zum Thema Robotik sowie das Public-Private Partnership euRobotics vor. Um das Marktpotential der Robotik zu heben und die damit verbundenen zukünftigen Forschungs- und Entwick- lungsfragen zu identifizieren, wurde eine Strategic Research Agenda für Robotik in Europa für die Jahre 2014 bis 2020 entwickelt. Es wird aufgezeigt, wo Österreich noch Bedarf an Forschung und Entwicklung hat, wobei diese Bestandsaufnahme in die Entwicklung einer österreichischen Robotics Research Roadmap einfließen wird, die von der Arbeitsgruppe Robotik der GMAR entwickelt wird.

Als Gastherausgeber dieser e\&i-Ausgabe möchte ich mich an dieser Stelle ganz herzlich bei den Autoren für die Beiträge zu diesem Themenschwerpunkt zur GMAR, bei den Fachbereichsleitern und Vizepräsidenten der GMAR, Univ.-Prof. Dr.techn. Martin Horn (TU Graz), Ao. Univ.-Prof. Dr.techn. Thilo Sauter (Donau-Universität Krems), Ao. Univ.-Prof. Dr.techn. Markus Vincze (TU Wien) und beim Geschäftsführer der GMAR, Dipl.-Ing. Peter Reichel, für deren Unterstützung sowie bei den Gutachtern für deren exzellenten Einsatz, zeitgerechte und professionelle Gutachten für alle Fachbeiträge zu erstellen, bedanken.

Wir als GMAR hoffen, mit diesem Heft einen Einblick in die Kompetenzen und das Portfolio der Gesellschaft für Mess-, Automatisierungs- und Robotertechnik gegeben zu haben, und würden uns sehr freuen, wenn wir Ihr Interesse an der GMAR wecken konnten und es uns gelungen wäre, interessierte Personen und Betriebe zur Teilnahme in der GMAR zu motivieren. 